\title{
Spatiotemporal Analysis of Circulation Behaviors Using Path And Residing Time displaY (PARTY)
}

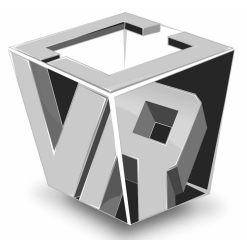

\author{
Kingkam Sookhanaphibarn, Ruck Thawonmas, and Frank Rinaldo \\ Ritsumeikan University, Kyoto, Japan
}

\author{
Kuan-Ta Chen \\ Academia Sinica, Taiwan
}

Editor: Zhigeng Pan

\begin{abstract}
Spatiotemporal data displayed in a spatial layout are not the best visualization for finding similarities of visitor paths and extracting pattems of visitor interest to placed items. A challenging problem is the visual analytics of cinculation pattems in varying layouts commonly found in a museum with many exhibition rooms. This paper proposes a layout-independent visualization approach to represent a visitor path and his/her time spent residing near the closest item. In this approach, we encode a time interval residing in an item boundary into a color-shaded line segment. Color shade is used as an indicator to the proximity distance to the nearest item. The length of a segment is in proportion to the total time spent in the layout. The time segment is placed in the row corresponding to its item boundary. A path of visited items is illustrated by connecting the time segments with vertical lines. The resulting visualization technique, called Path And Residing Time displaY (PARTY), enables users to find trends of circulation behavions in a consistent fashion regardless of the targeted layout. We demonstrate the effectiveness of PARTY on two datasets: one showing circulation behaviors of visiting styles in a 3D virtual museum and the other showing a flow of people escaped from an explosion in a building. PARTY is applicable for analyzing data in real and virtual spaces. While the focus of this paper is to apply PARTY to discovering circulation pattems in museums or art galleries, the utilization of this approach covers also visual analytics of customer circulation in a number of environments (e.g. convenient store, department store, World's Fair, etc.). PARTY provides useful information about the number of visitors to items, flow pattems, crowded areas, items not visited, and other aspects of visitor behaviors.
\end{abstract}

Index Terms - circulation behavior, layout-independent visualization, movement pattem, proximity distance, visitor trajectory, virtual environment, visual knowledge discovery.

\section{Introduction}

Visualization of spatiotemporal data is an interesting and challenging research area. Most visualization techniques using traditional twodimensional maps present the paths of and the time spent therein of visitors directly over spatial layouts. In this paper, we call layout-dependent visualization for any visualization technique using the spatial layout of a targeted area as its graphical background in visualization.

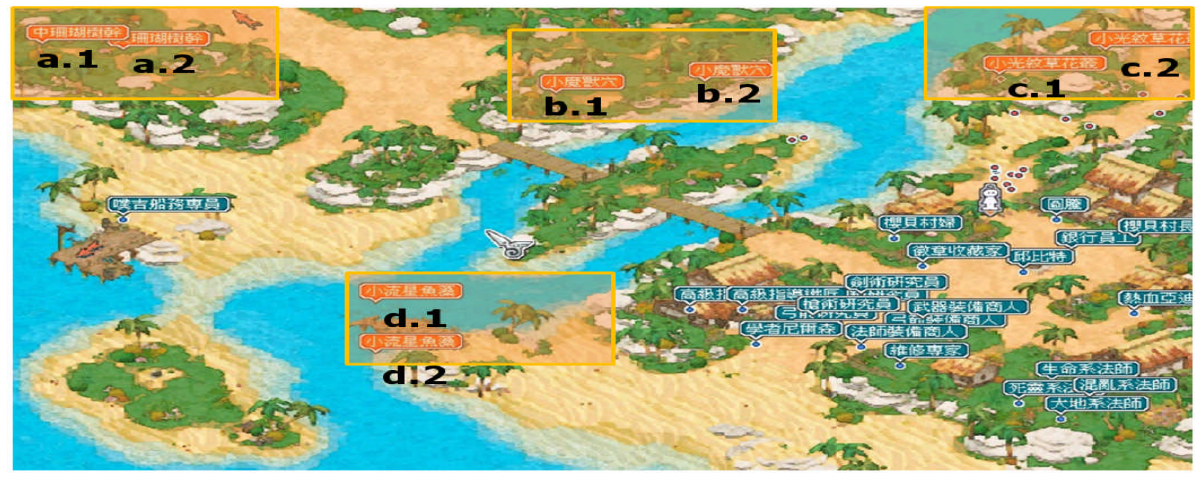

Fig. 1. Map of one island in Angel Love Online (ALO) where eight resources in four locations are distributed apart.

\subsection{LAYOUT-DEPENDENT VISUALIZATION}

Three challenging problems of layout-dependent visualization are as follows:

1) Understanding of the visualization results requires the familiarity with the targeted layout.

2) Examining of a set of selected items placed apart from each other is easily interfered by the other items placed in the layout.
3) Presentation of circulation patterns in the visualization is done in an inconsistent fashion.

The layout-dependent visualization approach burdens users by a cost of requiring them to recognize the layout of items and routes by themselves. Some additional symbols indicating the position and boundary of items as well as arrowheads indicating representative routes must be placed in layout-dependent visualization. However, these symbols and arrowheads conceal visitor traces from users. 
Such a situation, in which users examine items placed in different areas, is disruptive since a layout-dependent visualization must display all areas covering those items. Areas of no interest will enlarge the size of that visualization, and items and areas of consideration will be de-emphasized. If an item group of interest (denoted by orange rectangles) are located far apart others as shown in Fig. 1, the circulation patterns of players' avatars among the item group will be hidden away by the layout context.

A circulation pattern is defined as how visitors move within the space with respect to their common sequences of visited items. As a result, the visitor trajectories shown in Fig. 2 are considered the same circulation pattern whose visitor trajectory explores an exhibition following the same predefined route.
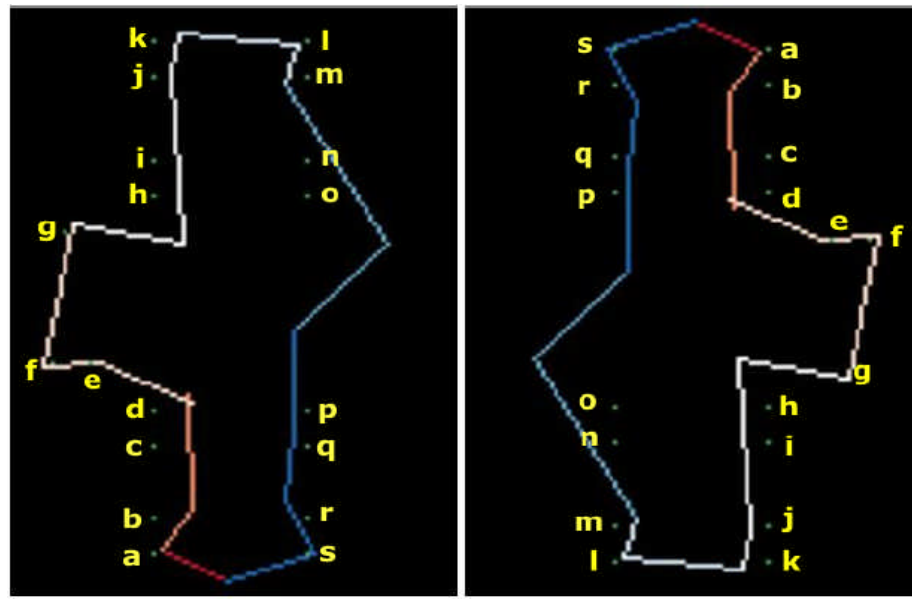

Fig. 2. Similar circulation patterns in observing a set of items a to $s$, i.e., the circulation ring starting with a left turn, in two different item layouts whose routes are colored from red to blue; (Left) Original; (Right) Different orientation.

Its beginning and ending segments are represented by starting with red and blue, respectively. However, visual presentations in varying layouts are substantially different in shape, size, and orientation as found in a number of environments such as convenient stores, department stores, World's Fairs. Those visitor trajectories are distorted by the shape and size of spatial layouts as well as the orientation of items therein.

\subsection{LAYOUT-INDEPENDENT VISUALIZATION}

A layout-independent display for visual analysis is a representation of the path and residing time of the movement data in circulation. The circulation behaviors of visitors moving through a place of interest, say a museum, are influenced by the items on which the visitors focus their attention. Thereby, taking for an example of museum case, we highlight a set of items located in a museum by representing them as the vertical axis, in which the item order follows the intended route developed by the museum curators. Instead of using a spatial layout of a targeted museum, for each item, we plot the time interval in a row corresponding to the item's boundary. We notice that each item boundary is related to the corresponding partition of the museum space. As a result, our visualization will transform a visitor trajectory to time series, i.e., a sequence of visited items. A merit of this data transformation is that of facilitating the analyzing and forecasting tasks of the circulation behaviors in the domain of time series.

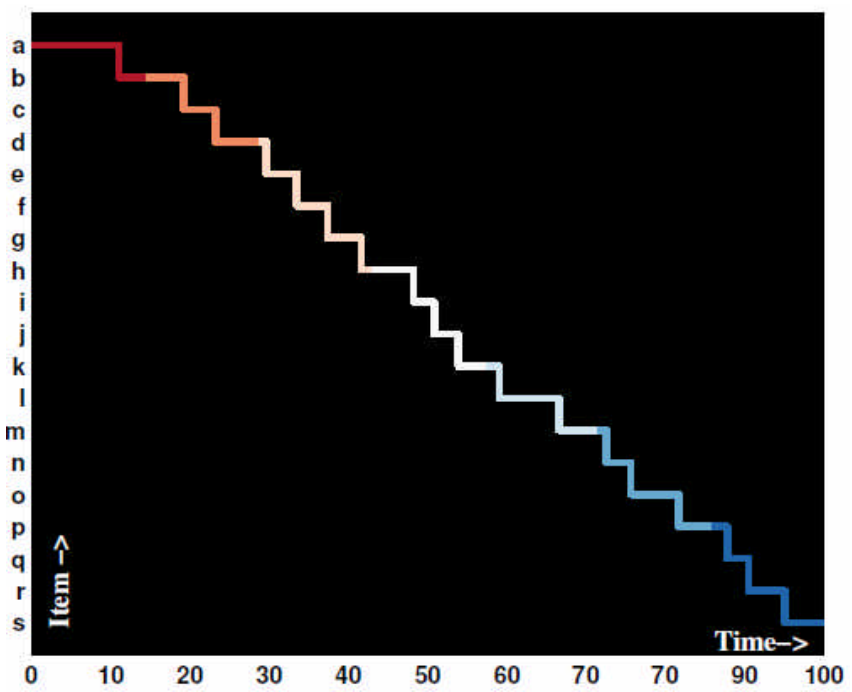

Fig. 3. PARTY produced the invariant visualization of the visitor path obtained from both the left and right routes of Fig. 2.

\subsection{OUR PROPOSED APPROACH}

In this paper, we introduce both local and global visualization approaches that are independent of the space layout, naming them Path And Residing Time displaY (PARTY). PARTY can be used to analyze the circulation patterns of movement data in a number of environments. Applicable analysis targets include visitors in an exhibition, people who evacuated during an incident, or virtual characters in virtual space. Figure 3 is a use of PARTY for presenting the visualization of a single visitor exploring a set of items in a museum.

We applied PARTY to three use cases where one is real data from an online game and the others synthesized data. The first one is a dataset of real users playing a popular massively multiplayer online game in Taiwan, named Angel Love Online (ALO), where the targeted map is shown in Fig. 1. The second one is a dataset of 36 synthesized visitors with the concrete definition of four visiting styles as addressed in [24]; this data set is generated in a virtual museum environment as shown in Fig. 1. The third one is a dataset of trajectories of 82 people in a building as shown in Fig. 4 who were escaping from an explosive; this data set is synthesized for the VAST 2008 challenge. 
The paper is organized as follows. Section 2 defines the circulation behaviors used in this paper as described by several researches related to analyzing and finding of circulation behaviors in museums and art galleries. Section 3 discusses related work in the field of spatial and temporal data visualization followed by varying visualizations of temporal data. Transformation from spatial data to proximities is introduced in Section 3 for a layoutindependent visualization. Section 4 describes design decisions of PARTY including the observation distance represented by the color appearance in our visualization and the observation range showing the boundary of each item placed in the spatial layout. Section 5 demonstrates the above mentioned applications in one of which PARTY is capable of dealing with more than 2,000 trajectories. Finally, conclusions and discussions are given in Section 6.

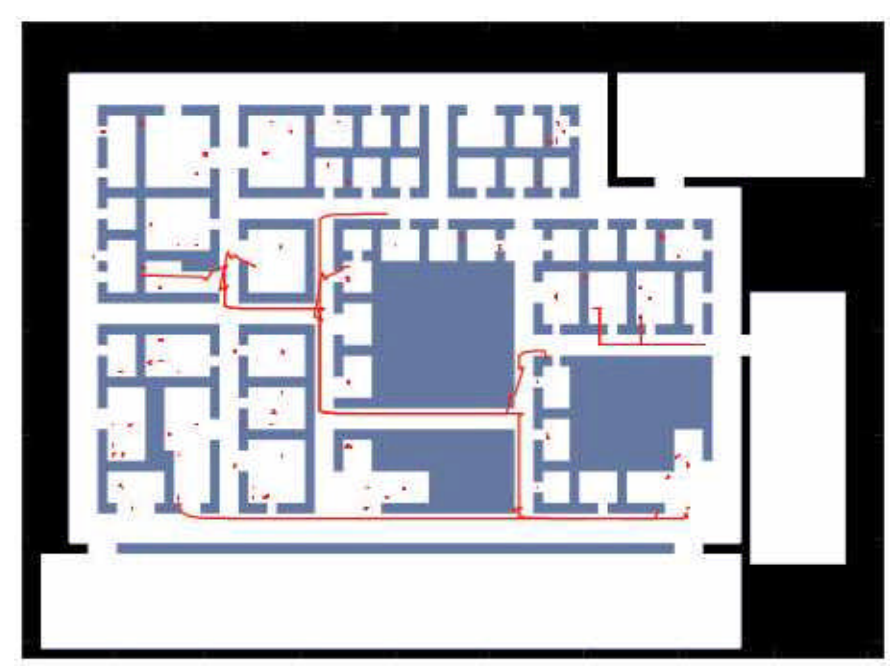

(a)

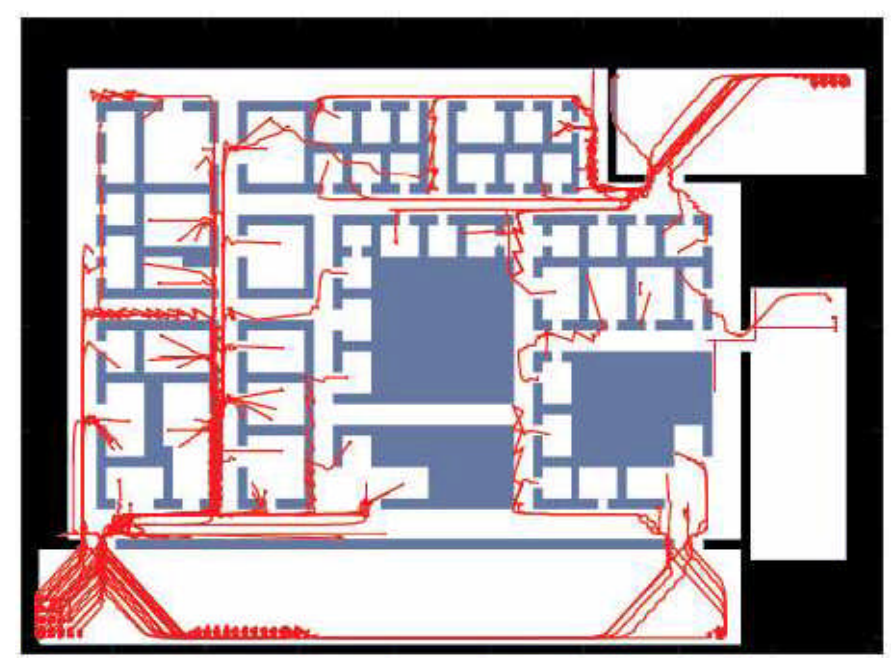

(b)

Fig. 4. Trajectories of people denoted by red lines in a building;

\section{Circulation Behaviors}

The analysis of circulation behaviors has received much attention in a number of researches on exhibition design. Exhibition design is likely to be successful if principles of circulation within exhibitions are applied to the design process. Visitors learn and are satisfied when the exhibition is designed with an understanding of the factors that determine circulation behaviors. Therefore, the visualization of visitor movements in an exhibition is a very useful tool for a space designer. Visitors in not only exhibitions but also other environments such as convenient store, department store, World's Fair are studied in the field of circulation behaviors.

In addition to a personal interest and preference of visitors, the factors of their movements relate to a spatial layout, a semantic relationship among items and social environment [6], [12], [16]. First, a spatial layout is an important attribute that an efficient layout must implicitly provide the path-finding information to visitors. Second, a semantic navigation is similar to a writing principle of story coherence. In an exhibition, the semantic navigation impact of visitor routes since their route can tell the narrative related to a sequence of visited/passed items. Third, visitors gather together along their journey following their leader as well as their friends/ relatives. These three factors result in the circulation behaviors of visitors.

Discovering the circulation behaviors was investigated in art galleries and a science museum, which their findings address direction of visitor path and their visit time. Associated with four visiting styles investigated in art galleries by Veron and Levasseur [24], the description of these styles is shown in Table 1. Tracing the trajectories followed by a visitor and identifying where the visitor stayed for more time, the authors [8] allows users to categorize a visitor (or a group of visitors) depending on the aforementioned visiting styles. However, their visualization approach does not present a sequence of visited items. 
Table 1. Description of the metaphor of four animals.

\begin{tabular}{|l|l|}
\hline Styles & \multicolumn{1}{|c|}{ Descriptions } \\
\hline Ant & $\begin{array}{l}\text { Visitors observe all exhibits in order or reverse } \\
\text { order by standing near those exhibits, and } \\
\text { there are no large differences in each exhibits } \\
\text { observation time. }\end{array}$ \\
\hline Fish & $\begin{array}{l}\text { Visitors spend their time in the center area far } \\
\text { from all exhibits. }\end{array}$ \\
\hline Grasshopper & $\begin{array}{l}\text { Visitors spend variable time to observe differ- } \\
\text { ent exhibits and ignore the rest of them. }\end{array}$ \\
\hline Butterfly & $\begin{array}{l}\text { Visitors visit almost all exhibits but their } \\
\text { spending time at each exhibit is less regular } \\
\text { than those of the ant visitors. }\end{array}$ \\
\hline
\end{tabular}

In such a science museum, the authors [15] retrieved the following five main typical patterns: a) directly going to the landmark area, b) going around and staying in the robot area, c) went around backwards, d) visited at every place, and e) stayed for a long time. Based on their clustered patterns, the authors emphasized on a sequence of visited items and the residing time at their visited items. The authors emphasize the clustering methods using spatial partitioning on the layout of a science museum. They analyzed the visitor trajectories in high dimensional space that is not understandable for human visual ability.

Since visitor movements are navigated by items arranged in space, the important information in consideration of the circulation behaviors is time intervals of the visitor trajectory residing near the items. In this paper, we transform the visitor trajectory into the sequence of this time intervals from his/her starting to the ending points. The circulation information such as a sequence of items in proximity to the trajectory, a duration time residing in each item, and fluctuations in the movement direction cannot be presented by the traditional layoutdependent visualization approaches of the movement trajectory.

\section{RELATED WORK}

This Section is organized to explain the motivations for developing PARTY. We review techniques dealing with visualizing the visitor trajectories mapped on a spatial layout and describe the approach and application of proximity-based visualization where a visitor path and the corresponding time spent are presented in a 2D dimensional view.

\subsection{VISUALIZATIONS OF VISITOR TRAJECTORIES}

A simple presentation of a visitor trajectory containing temporal and spatial information is a set of colored polylines overlaid on the place map in which visitors traveled. The color shades and polylines enhance a local visualization of a visitor trajectory in [11] for exploring the corresponding time spent. A path is in the form of connecting segments from red to blue. The session is equally divided in time into 7 periods in the ascending order from red to blue. The color of a particular segment indicates the passage of time. Its visualization is complicated in such a case shown in Fig. 7 (a).

To explore the trajectories of multiple visitors, Börner and Penumarthy [5] plotted the trajectories as colored polylines that interconnect discrete positions. They then used distinct colors distinguishing between different visitors. The aggregated trails were shown in their visualization based on the concept of a leader and her/his followers. Their group movement can be visualized by determining the coordinate of the centroid of all group members at a time unit and then drawing the connecting lines of these centroids.

Exploiting the color intensity illustrates either the time spent or the traveled area as proposed by Chittaro et al. [8]. First, the time spent visualization uses a color intensity to indicate how much time was spent by visitors in each area of the targeted layout. It takes into account the speed of entities, because the slower they move, the longer time to pass through an area is. Second, the traveled areas visualization uses a color intensity to represent the frequency of visitor passing through each area of the layout.

Visualization of frequent traveled regions [15] is conducted by spatial partitioning of all visitor trajectories. A k-mean algorithm was applied to the trajectory data to divide the space into $\mathrm{k}$ areas. Therefore, a more crowded point is likely to be the center of a cluster, and an uncrowded point is likely to be the periphery of a cluster. As a result, the targeted layout is automatically partitioned into a number of regions based on the global information. Afterward, each visitor trajectory can be illustrated by painting a passed region with a shade. A distinct shade present different time spent. The darker color indicates that a visitor staying longer in a particular region while the brighter one shows the shorter residing in this region.

In addition to the aforementioned $2 \mathrm{D}$ visualization approaches, many visual analytics of visitor trajectories [1], [2], [9], [23] provide in 3D display by using the human geographers' technique of space-time paths. These approaches are called "Space Time Cube" that the cube consists of a representation of the geography (along the $\mathrm{X}$ and Y-axis) and the time (Z-axis). The space time-paths are an instance in the cube. Although the $3 \mathrm{D}$ visualizations are able to include a geographical map where items are indicated by symbols and colors, the 3D displays introduce ambiguity and are hard to navigate. 


\subsection{VISUALIZATION IN OTHER PRESENTATIONS}

Researchers in information visualization have attempted to investigate the best presentation for human visual analytics. There are varying metaphors of pencils, a spiral, a wheel, and rings. Those techniques are briefly described below.

First, the metaphor of a pencil is used in the historical events [6], named Lexis Pencils. Each of its faces represents a different variable, and its length means the length of the event history. Calendar time runs along the length of the pencil. The size, value, texture, color, orientation, and shape of the face can indicate the variables' values. Categorical variables can be represented by a set of colors, textures or patterns. Such a case of the life history of a married couple can be represented by Lexis Pencil. Time runs from left to right, starting at the date of marriage and finishing at the survey date. Each face of the pencil represents a different variable. The top face represents the employment history of the wife, the middle face that of the husband and the bottom face the age of the youngest child in the household.

A view of Lexcis Pencil illustrates the temporal data. Afterward, these Lexcis Pencil can be embedded into a geographical map in order to representing spatiotemporal data. It is an advantage of the 3D visualization that increases the possibility of integrating additional visual information into the representation. However, the other visualizations below are designed in two dimensions.

Second, the spiral visualization technique [7] displays serial periodic data. The data have a serial dimension that exhibits periodicity. The data continue serially from day to day, month to month, and year to year. The data represent along a spiral so that the visualization highlight serial attributes along the spiral axis and periodic ones along the radii. However, this visualization is hard to design for the spatiotemporal data.

Third, the Time-Wheel technique [22] is to present the axis of reference (time in this case) in the center of the display, and to circularly arrange the depending axes around it. A single colored line segment makes a connection between a time value and the corresponding variable's value. From each time value, a colored line segment is drawn to each variable axis on the display. This approach aims to visual the time-dependent multivariate data. A shortcoming of this visualization is the number of variables. At most 15 axes can be analyzed by users familiar with visualization and data analysis. Efficient extraction of salient features becomes difficult when visualizing more than 10 variables depending on the axis of reference.

Last, Growth Ring Maps [3] are recent spatiotemporal visualization from sensor logs. This technique aims to reveal the behavioral differences of wild-type/transgenic and male/ female mice. Their behavior patterns take into account of the spatial and temporal information. The visualization use colors distinguishing places. The ring properties including its size and color gradient represent the number of visits and a time line, respectively. This visualization focuses on the growth pattern of visits over the time but discards movement paths.

\subsection{VISUALIZATION WITH PROXIMITY DISTANCE}

Using a proximity distance introduced by [10] is an alternative approach to visualize visitor trajectories in a fashion regardless of the spatial layout. A proximity data is a distance between a visitor position and a given reference location. A visitor trajectory is transform into a collection of the proximities by the Euclidean distance metric. The advantage of using this approach is to view spatiotemporal information in a 2D display by reducing spatial information from $2 \mathrm{D}$ to $1 \mathrm{D}$ and retaining time in the other dimension.

This visualization approach focuses on the proximity distance to a set of reference points versus time. As used a single fixed reference point, the proximity distance does not distinguish regions A from B as shown in Fig. 5. To solve this problem, multiple reference points are applied as described by [10]. The authors do not limit their proximitybased visualizations to a single distance dimension. It means that the distance to multiple points of reference can be defined. Since their visualization is in a form of a $2 D$ display, the multidimensional proximity data point must be projected to a single dimension that the other dimension is retained for time. However, the projected data is difficult for humans to understanding.

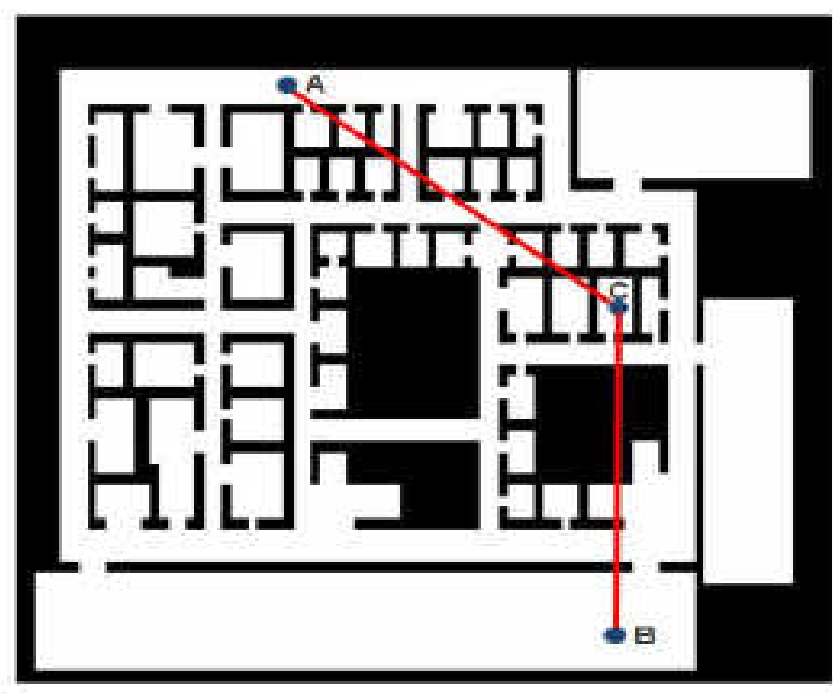

Fig. 5. Regions A and B having the same proximity distance from a fixed reference location $C$.

In our paper, we use a path of residing regions rather than proximities versus time. The decomposed regions in a spatial layout are systematically fixed by items of interest, for example, we use the boundaries among items residing in a museum as regions. It is possible to specifically define regions by users in order to find the desired solution, for example, we divide the layout of a given building floor into small blocks depending on their investigation questions. A path of residing regions is represented on a vertical axis of PARTY. 


\section{Design Decisions}

Designers of museum space and online games as well as investigators of a terrorism situation require several types of information for examining the circulation behavior of visitors. These include (1) locations, regions, or item boundaries, (2) a time interval in each visited region, (3) a path of visited regions, (4) information about multiple visitors residing in a particular region or time spent therein, and (5) a degree of their interest depending on applications. All of these data types are computed from (a) a log file of visitors' positions including $\mathrm{x}-\mathrm{y}$ coordinates and time and (b) the map of a museum or a floor plan including the item locations or the room positions.

The design of PARTY aims to represent three dimensional entities, i.e., a time unit, a visited item, and a visitor. The horizontal and vertical axes of PARTY represent time and items which the visitor resides within the close proximity to the items. A stack of visitor stripes is put inside an item block (row). As shown in Fig. 6, there are five items of interest and three visitors. The stack of three visitor stripes places inside each item row. The order of the three visitor stripes is consistent thorough the five item rows.

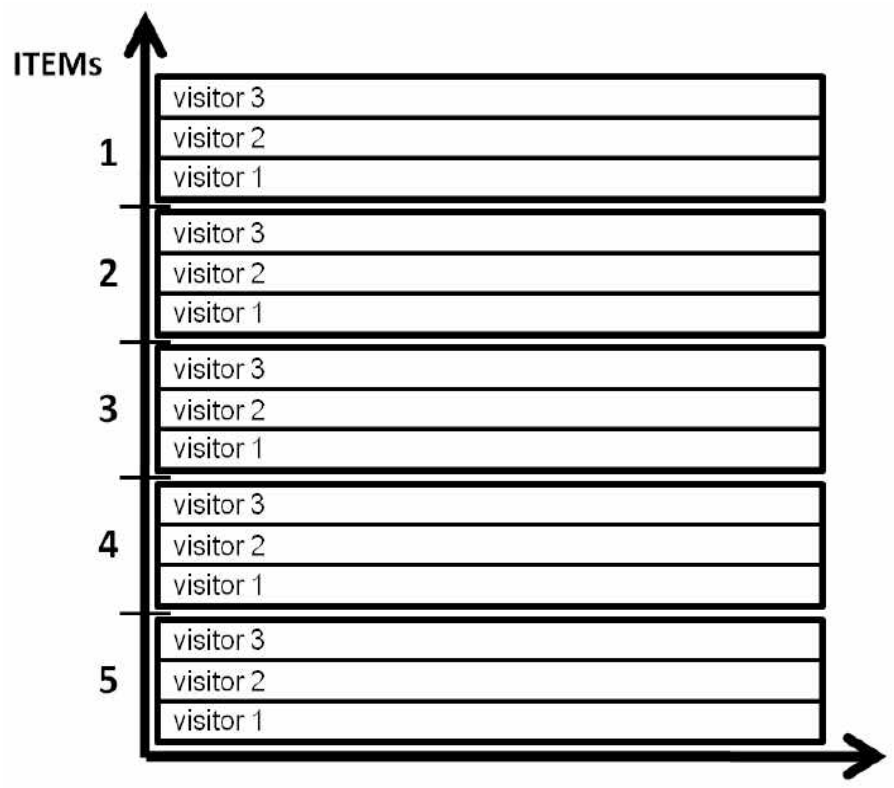

TIME

Fig. 6. Structural design of PARTY.

We arrange the visitor stripes in every stack in the same order and rank them by the similarity among their circulation patterns. Then, we use a hierarchical clustering technique for finding the similarity of all pairs of visitor paths. To handle hundreds of visitors, the representative of each group of visitors can be used rather than a single visitor. The representative is derived from the generalized median defined as the visitor path having the nearest distances to the others in the same group.
Besides a path of nearest items versus time, the proximity distance to the nearest item can be displayed in PARTY as a degree of visitor's interest to each exhibit item. A displaying color is computed using the observation distance and range based on the locations of all items. For example, given a visitor trace as shown in Fig. 7(a), Fig. 7 (b) represents the visitor trace as our observation-based time series. The visualization in Fig. 7(b) consists of the horizontal axis corresponding to the visit time, the vertical axis indicating an item belonging to the observation range at a particular time, and the shades of red showing the observation distances. This visualization is produced by using the observation distance and range in the application of a 3D virtual museum in Section 5.1.

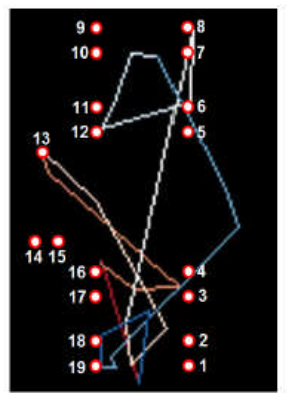

(a)

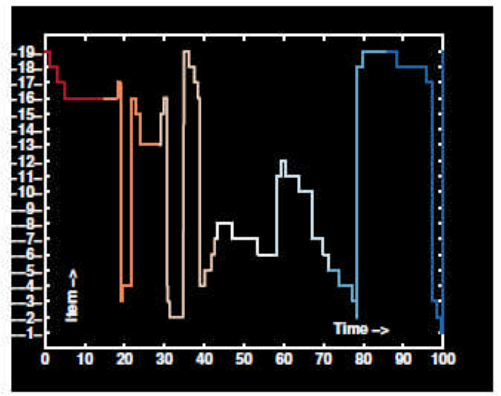

(b)

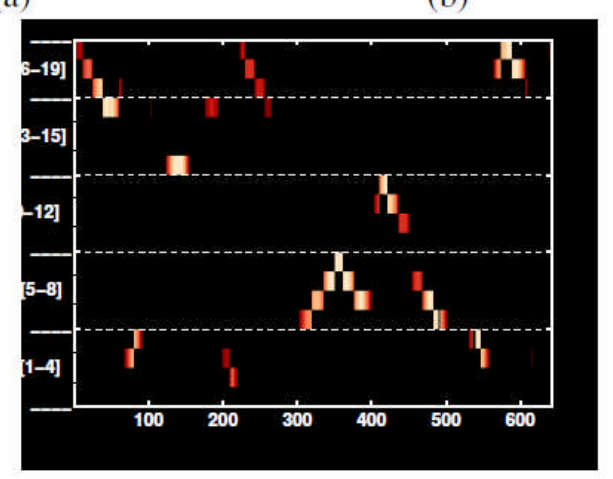

(c)

Fig. 7. Transformation of a single visitor trace for PARTY; (a) Representation of a visitor trace tracked in a $3 D$ virtual museum; (b) PARTY of a single visitor that represents a path of visited items ( $Y$-axis) and his/her spending time; (c) Representation of the visitor interest to items by using color shades from brightness to darkness as highest to lowest degrees.

\subsection{VISITOR INTEREST AS OBSERVATION DISTANCE}

The consistent color scheme using ColorBrewer's nine level sequential shades [11] is applied throughout PATRY. The shades from the brightest white to darkest red present the decreasing degree of visitor interest. The interesting 
degree is transformed from the visitors' movement approaching to each item. It implies that visitors pay attention to an item whenever these visitors move closer to that item. In our visualization, the colors from darkness to brightness indicates that visitors are approaching to an exhibit item of interest.

With respect to the physical properties of varying exhibition items, the best observation area is not always nearest to those items. The best observation areas, which are colored by the white color, are simply defined by exhibition designers or curators in charge. The other positions far from the best area are the second three brightest shades. These color shades are mapped to the farther distance from the best area. The darkest red shows that the visitor is approaching to or passing that exhibit. Last, the black indicates that a visitor does not approach to that exhibit.

An example is shown in Fig. 8 (a), which is a map of RDAP (Ritsumeikan Digital Archiving Pavilion). RDAP is a virtual gallery displaying the kimono textiles of 19 objects. At all locations, kimono textiles are hung against the wall; therefore the brightest shade, white, is displayed near the border except the right wing as shown in Fig. 8 (b). Inversely, the hallway and the middle area are illustrated with the darkest shade of red. The black color is used for area outside the gallery.

\section{A. Observation Range as Voronoi Diagram}

In our visualization, shading colors against time present the direction approaching to/ leaving from a particular exhibit item. For this purpose, the observation range of each item is calculated by using Voronoi diagram. The decomposition of exhibition space is determined by distances to a set of exhibit items in the space. For example, the layout of RDAP is partitioned into 19 regions derived from the locations of items as shown in Fig. 9.
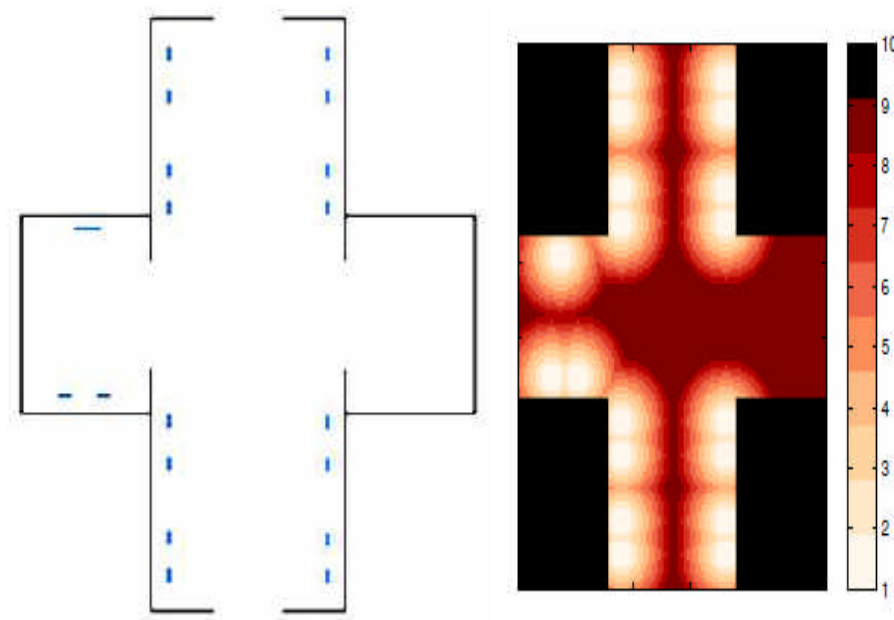

Fig. 8. Example of observation distance map; (a) RDA P map; (b) Observation distance map.

\subsection{TIME INTERVAL IN AN X-AXIS}

PARTY represents a whole route of a single visitor spending his/her time in a targeted environment. A time interval represented by PARTY is a relative value with a relationship of his/her total time. In other words, a time unit along the X-axis of PARTY indicates a percentage of the total time spent. Therefore, PARTY can analyze log files of any visitors regardless of their real visit time.

\subsection{ITEM ORDERING IN A MAJOR Y -AXIS}

The order of items can be automatically arranged by using a conventional Genetic Algorithm (GA) with the objective function that minimizes the total length of all paths drawn on PARTY as well as the number of crossing lines among paths. GA starts with an initial population where each chromosome represents an order of items. The best ordering of Items will result in PARTY with the optimal total length of all paths and the least number of crossing lines.

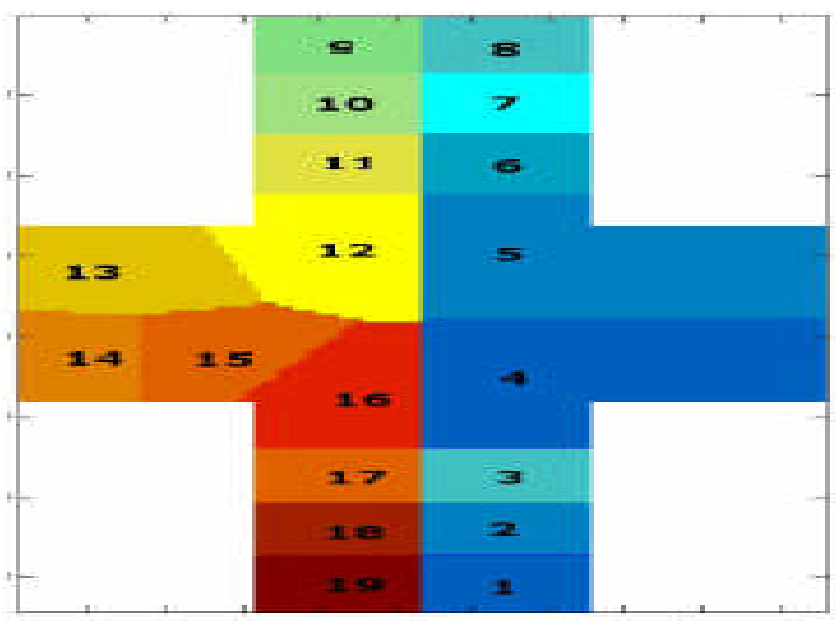

Fig. 9. Item boundaries derived by using Voronoi diagram in accordance with Fig. 8.

\subsection{STACK OF VISITOR TRACES IN A MINOR Y - AXIS}

PARTY represents all visitors trajectories as a set of stacks each of which provides information about the residing time intervals corresponding to an item. The visitor information in a stack is represented as a stripe. For example, Fig. 10 represents the stack of the first item row showing visitor 1 resided near item 1 at the beginning and end of his/her route. Visitors 2 and 3 have the same visiting pattern at item 1 where they stopped near it for a long period of their visit time. The stack representation allows users to visually analyze the visitors' trajectories in aspect of global and local visualization. 
ITEM \#1

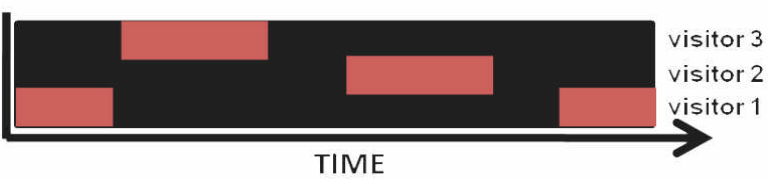

Fig. 10. Stack of three visitor stripes in the first item row where the red color denotes their residing time intervals near item 1.

All stacks in PARTY have the consistent order of visitor stripes. The ordering can be in accordance with the dissimilarity matrix that is the distance between every pair of time series data that are derived from visitor trajectories. Otherwise, users can explicitly specify their order. There are many techniques to calculate the dissimilarity matrix. In this paper, we compute the dissimilarity matrix by using the dynamic time warping and the Euclidean distance and then sort the time series data in an ascending or descending order.

\subsection{Group-Based Stack of Visitor Traces}

The structural design of a stack of visitor stripes allows users to visually find similarities of visitor paths and residing time intervals. This can enhance PARTY to handle a large number of traces. The discovered trends can be represented as a new definition of the visitor stack. Unlike the stack of all visitors, a group-based stack shows only representatives of similar time series data while PARTY in a fashion of the all-visitor-based stack is shown in Section 5.1 and 5.2. The width of stripes in the group-based stack is proportional to the total number of visitors. The wider stripe, the larger number of similar time series are found in this cluster.

\section{Applications}

\subsection{APPLICATION TO A 3D VIRTUAL MUSEUM}

This section presents an application of PARTY analyzing the avatars' trajectories and finding trends of circulation behaviors in the 3D virtual museum, RDAP. RDAP, owned by the Global Center of Excellence in Digital Humanities Center for Japanese Arts and Cultures, of Ritsumeikan University, is built in Second Life (SL). An objective of RDAP is to disseminate Japanese costumes, Kimonos, preserved in a digital achieving system. We synthesize the visitor trajectories based on the metaphor of the four animals as mentioned in Section 2.1. The total number of synthesized trajectories is 36 .

As shown in Fig. 11, PARTY displays two typical pattern routes: one starting from the top left to the bottom right and the other starting from the bottom left to the top right. For example, one typical route 1 (denoted by a gray curve drawn) starts from items 4 followed by the other items along the $\mathrm{Y}$ axis with ending at 9. Following route 1, visitors walk slower in the first half and then the visitors hurries up in the second half. The typical pattern routes imply that the re-arrangement of the item order in the museum can a) avoid visitors to miss some importance exhibits and b) encourage visitors to observe all exhibits in order.

References [20], [21] review a number of existing virtual museums and galleries in SL where displaying objects/exhibits in virtual worlds can be dynamic and its rearrangement occurred frequently. With PARTY, the designer and developer of a gallery, for example, can promote a new item by putting them beside a popular item. They also would like to arrange a set of exhibits to avoid a user missing some items unintentionally.

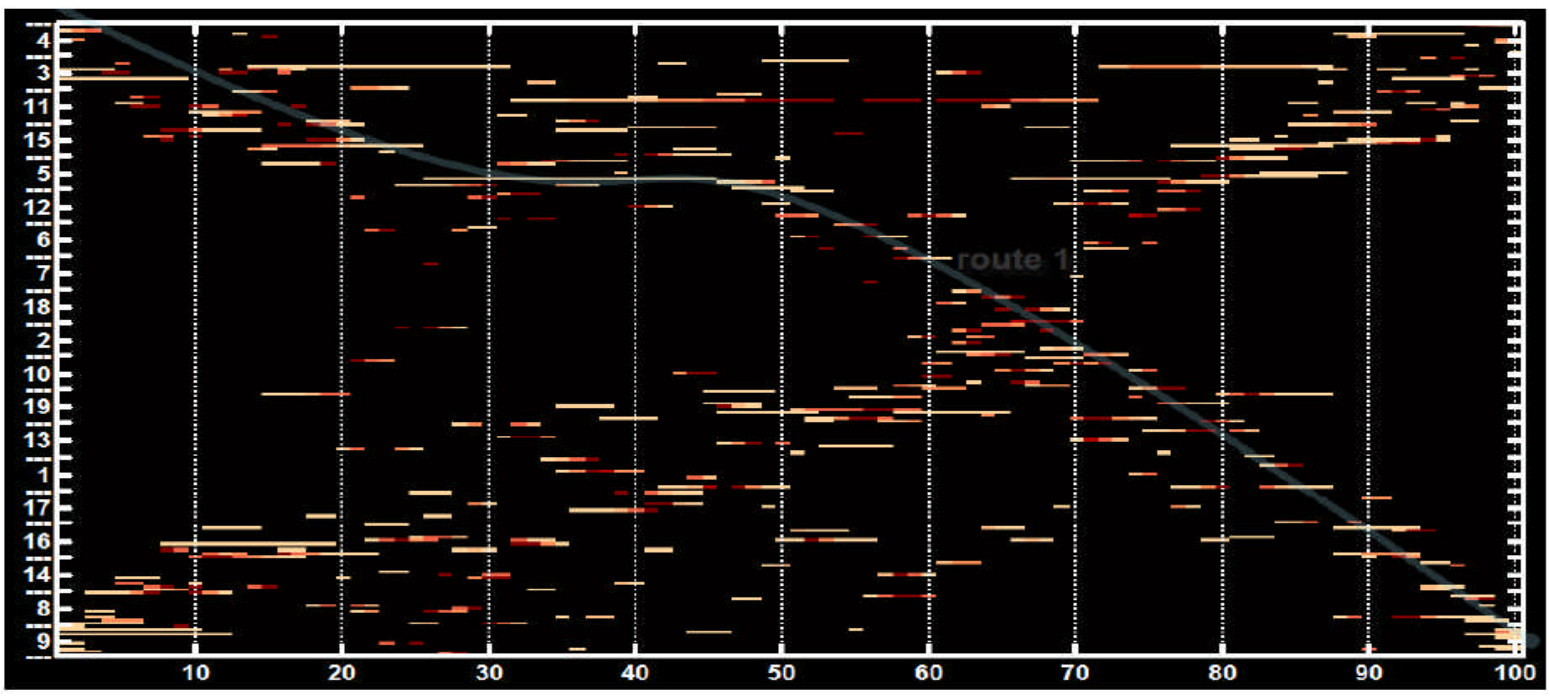

Fig. 11. PARTY of the museum data set showing degrees of visitor interest, most common time periods of visited items, and also the flow of visitors for one journey session in the

RDA P museum the layout of which is shown in Fig. 8 and 9. 


\subsection{APPLICATION TO ANGEL LOVE ONLINE}

For the second use case, PARTY presents the most typical circulation behaviors derived from the ALO data set []. ALO was developed by UserJoy Tech. Co. Ltd, a leading game designer and publisher in Taiwan. Our target layout is the most popular island because of its inclusion of the resources for increasing the fighting skill of players. There are four different types of resources located separated in the map as denoted by $\mathrm{a}, \mathrm{b}, \mathrm{c}$, and $\mathrm{d}$. The landscape of the island is two sides connected via a small strip of land in the center surrounded by water. The sea (denoted by black color in Fig. 12) is an area players cannot access. In the data set, there are 122 unique players with 2,231 total sessions that was collected from this map for about 70 hours.

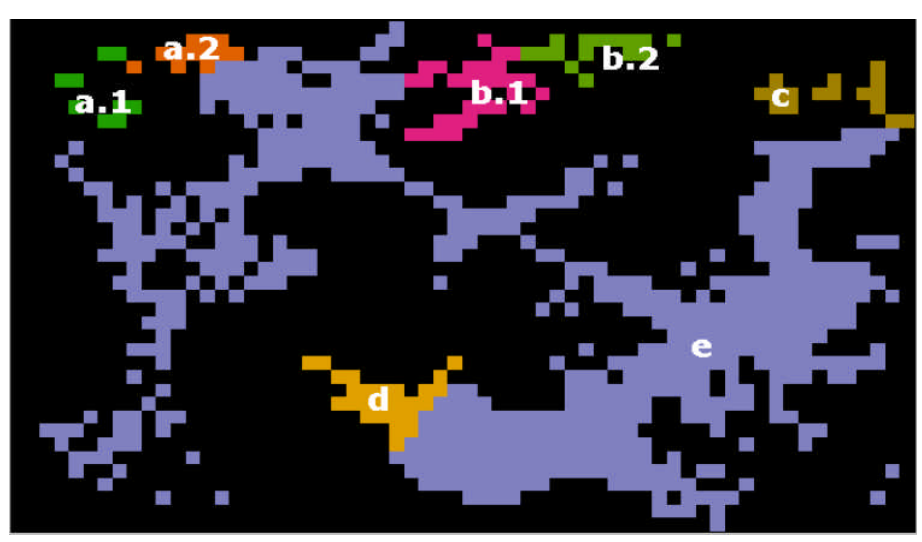

Fig. 12. Decomposition of the ALO map associated with the resources as shown in Fig. 1.
At the beginning, we decomposed the map into the main regions by using the ISOMAP technique [19] to partition the space so that any distances among the connected locations were considered as the shortest path in the Euclidean distance set. This decomposition technique results in seven regions corresponding to the locations of the resources as shown in Fig. 12. Then, all trajectories are transformed into time series data for the PARTY representation as explained in Section 4. PARTY as shown in Fig. 13 uses the strategy of a group-based stack of visitor traces as explained in Section 4.6. Using PARTY, users can explore the following information

1) Typical circulation patterns of players collecting resources

2) Circulation patterns residing in the particular location for seeking resources

3) Most popular locations for collecting resources

A typical circulation pattern is the best representative of a data cluster where the representative must be real data from the whole data set itself. The representative must be nearest to the others. From Fig. 13, we show a use of group-based stack for the six most typical patterns among the locations of the six resources as shown in Fig. 1. Six colors are used for indicating trace patterns that resides in one particular resource longer than the other resources.

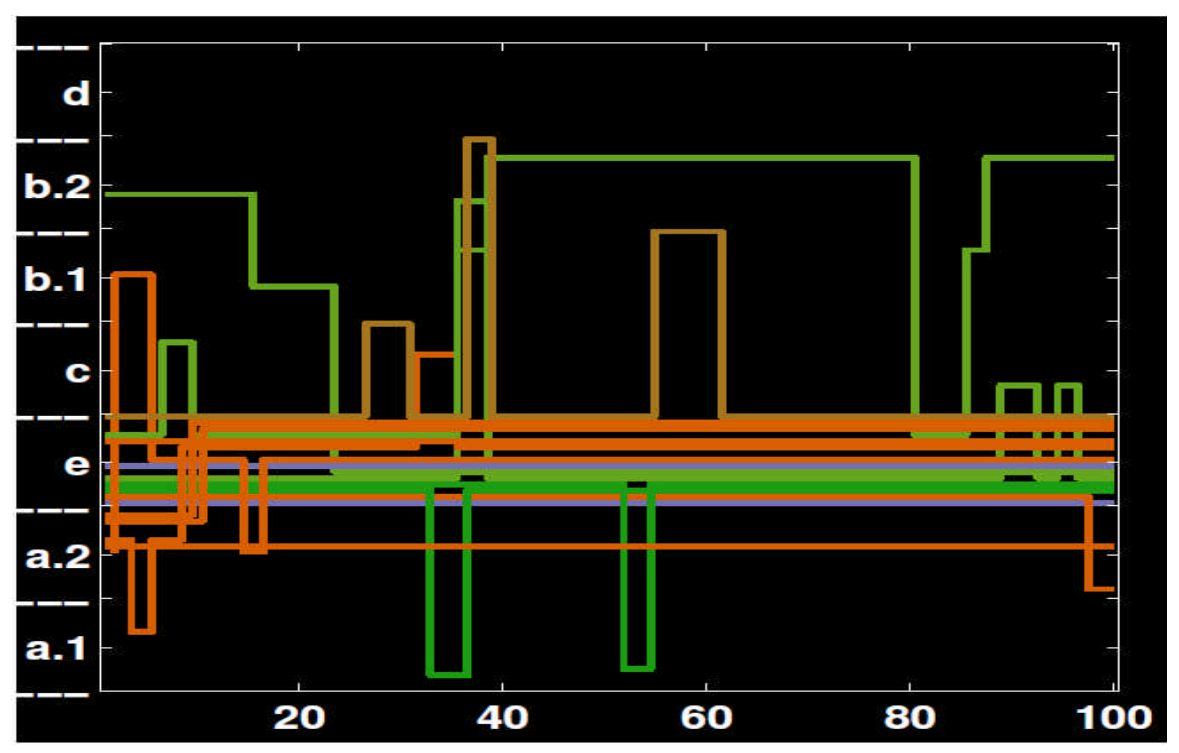

Fig. 13. PARTY of the ALO dataset showing the most typical circulation patterns among the locations of the six resources as shown in Fig. 1 . 
Each typical pattern indicates a most common behavior of characters in a game and can be used to verify the game story designed by a game developer and to explore a network usage at particular area. Generally, a game company can observe and analyze game-play, environment, storyline, and characters before a new game update will be released. With an interaction feature, user can select a particular typical pattern or a group of patterns for comparison.

\subsection{A PPLICATION TO AN EVACUATION SITUATION}

Evacuation patterns when an explosion occurs in an office building are considered to be another type of circulation patterns. Exits or safe areas in the building are the destination in this situation. For the investigation of an incident, an analyst uses PARTY for:

1) Describing flows of evacuating people through safe areas

2) Identifying when and where the explosion occurred

3) Finding potential suspects or witnesses to the event

We apply PARTY to the evacuation data set from the IEEE VAST 2008 Challenge [12], [8]. Such a situation is that the detonation of an explosive was set off at a Department of Health (DOH) building, Miami, Florida. All people in the building wore badges enabling their location to be recorded during the time of the incident. The data recorded is approximately 15 minutes and contains 82 people traces. The coordinates are mapped to a $91 \times 61$ grid space as the map is shown in Fig. 14.

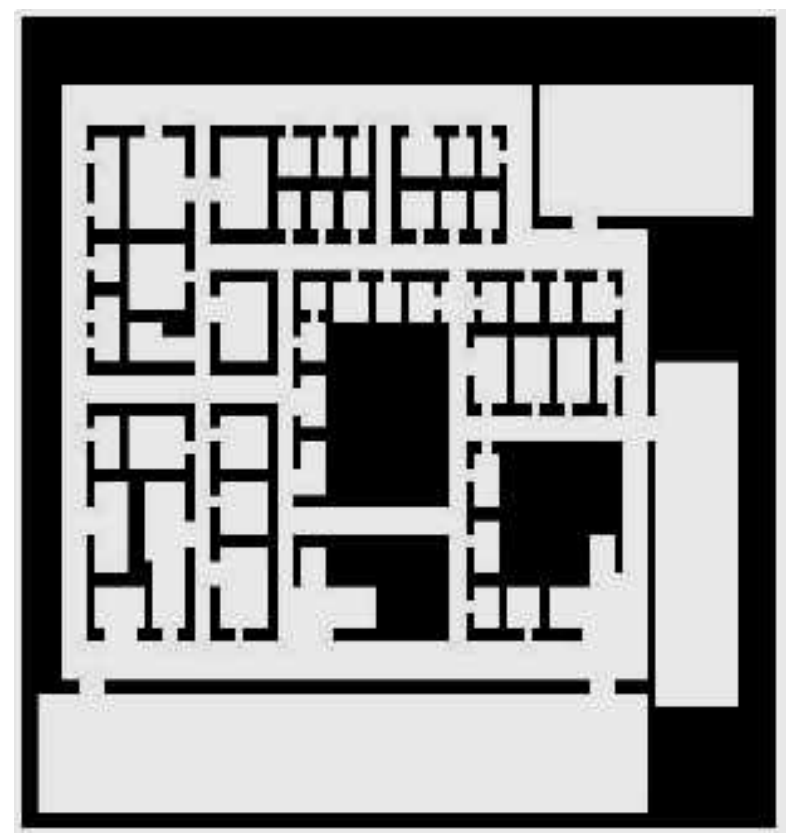

For analysis of evacuation patterns, the decomposition of regions appearing on PARTY is systematically and recursively computed as follows. The recursive decomposition can proceed in a particular region for discerning movements within it. We decompose the $\mathrm{DOH}$ map into 16 regions consisting of three safe regions, two walking area outside the rooms, and 11 blocks of rooms inside the building.

Our numbering procedure of decomposed regions is derived from the designer's idealized paths. In an evacuation situation, it is supposed that the flow direction of circulation patterns is a straight line to the nearest exits. For the sake of simplicity, two main evacuation patterns are considered as (a) flow to the green exit and (b) flow to the violet and orange exits. The flow to the green exit is assigned to the first number 1; the others are set to the two last numbers 15 - 16. Then, the walking paths are divided into two regions: left and right parts.

Basically, most of people on the left part escape to the green exit; therefore, the left region of the walking paths is assigned with number 2 . In the same manner, the right region is set to number 14 . For all blocks inside the building, the proximity from a block to the nearest exit is used for the numbering procedure.

After the decomposition and numbering procedure, PARTY in Fig. 15 shows the evacuation patterns of people moving from their location before the incident to the exits. Green, violet, and orange lines confirm the people who were able to escape from the explosion thorough exits 1,15 , and 16 . The other lines colored with

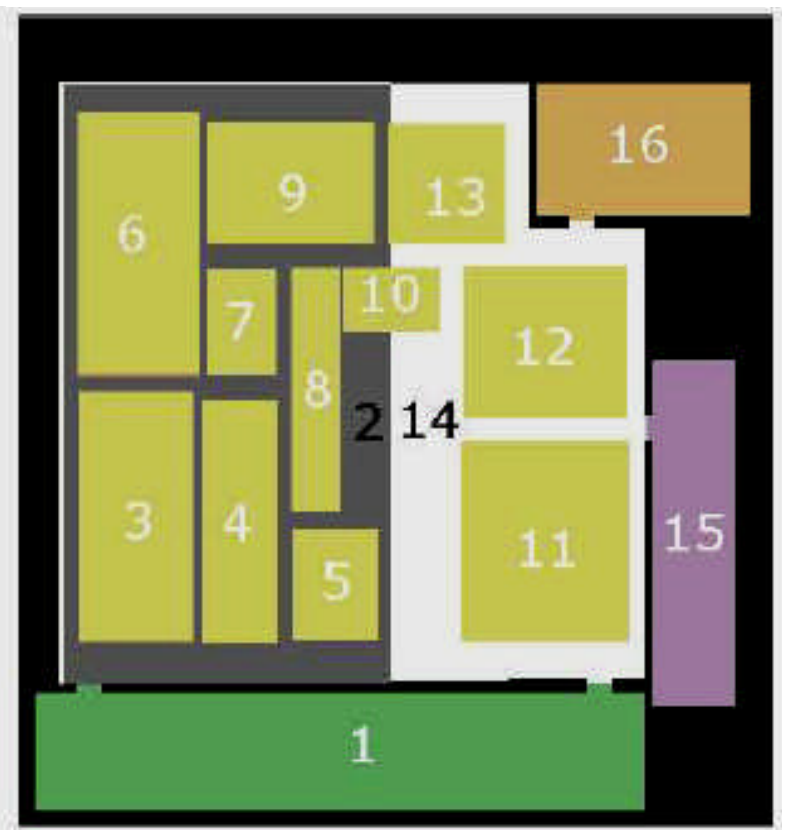

Fig. 14. Building layout and its decomposed regions; (a) Binary image of the building layout; (b) Decomposed regions by using a recursive partition. 
yellow show that a number of people were stuck inside. Visual analytics of the evacuation patterns by using PARTY can discover the following facts:

1) The majority of evacuation patterns were flows to exit 1 followed by exit 15 and 16, respectively. These are easily identified by comparing the width of stripes at the ending time.

2) People were stuck in regions 10,12 , and 13 as well as in the hallway regions 1 and 14 .

3) Most people left for the hallway regions 1 and 14 at time interval 381-480 second as seen by the width of dense vertical lines.

4) The approximate explosion time happened 10 seconds before they arrived the hallway regions by second 381 as noticed by the beginning of the dense vertical lines suddenly flowing through either row 2 or 4 . Therefore, The incident may happened by 371 because PARTY represents the residing regions where visitors have already arrived.

5) The suspected bomber was detected from his/her movement before the incident.

6) The location of bombs was possible in either regions 11 or 12.

7) There were bottlenecks in regions of walking paths that caused people to be still inside the building at the ending time.

\section{Conclusions and Future Work}

In this paper, we propose PARTY which is a 2D visualization of the residing time intervals and regions of multiple visitors as well as the visitor paths. Our main goal is to design 2D layout-independent visualization for visual analyzing circulation behaviors commonly found in a number of environments and situations. Through the three applications, we showed how PARTY supports visual analysis of visiting styles and trends in a 3D virtual museum, a game online and investigation of an explosion incident in an office building.

In the first and second applications, PARTY is beneficial to visitor as well as game player satisfaction and comprehension of items being observed. The main beneficiary of PARTY is a person who is in charge of efficient design of space. For example, curators must design an efficient exhibition with the understanding of the factors that determine circulation behavior. For this purpose, PARTY of visitor traces in exhibition space represents the following information:

1) Sequence of observed items along their visit time

2) Observing time and position at each item during their visit time

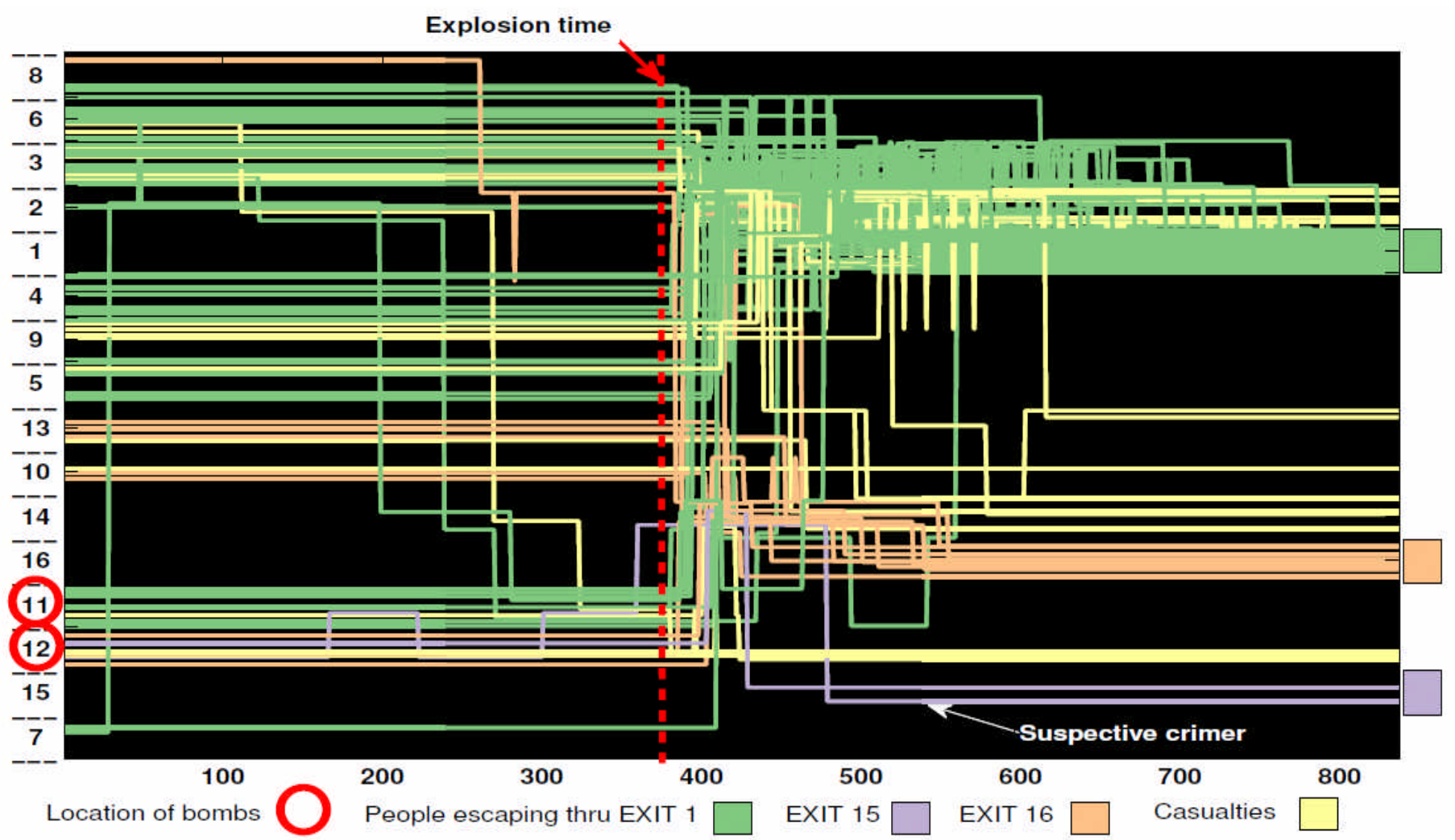

Fig. 15. PARTY of 82 visitor trajectories where lines are colored following their destination; Four colors are used for discerning between the visitors moving through exits, numbering with 1, 15, and 16, and the others staying inside the building. 
3) Comparison among multiple visit traces

4) Trend of visiting patterns within a particular time period

5) Majority of visiting patterns in given exhibition space

In the third application, PARTY is a power visual analytics tool to assist officers to conduct a comprehensive investigation after the incident. The main view of PARTY allows its users to answer important questions such as:

1) What are the typical evacuation patterns?

2) Which individuals might be suspects?

3) Where are locations of explosive setting devices?

4) When did the explosion incident occur?

5) What is the approximate number of casualties?

Moreover, PARTY is a very useful tool for analyzing customer behaviors. While there have been very high competitive business nowadays, an interesting question in order to gain more profit is like: How many Nordstrom shoppers also stop at Starbucks? How long do most customers linger in Victoria's Secret? Are there unpopular spots in the mall that aren't being visited? It is possible to collect the anonymous data from people cell phones. It can follow shoppers' paths from store to store. While U.S. malls have long tracked how crowds move throughout their stores, this is the first time they've used cell phones. But study tracking people via cell phone raises privacy issues. This paper, therefore, used only a data from the virtual worlds and synthesis data because.

There are three issues we consider for future work. First, we have described that the best observation location may not be the nearest position to an item. We plan to employ the visual analytics approach to find the best observation location introduced in [17]. Second, we have described the decomposition approach of a spatial layout into a number of regions when we applied PARTY to the evacuation incident. We will adopt the dynamic space division based on the quadtree representation addressed in [18] for the automatically recursive partition of the targeted layout. Third, we plan to enhance PARTY functionality with the recommendation approaches, such as predictions for the next items to be observed by a visitor in museum space.

\section{REFERENCES}

[1] G. Andrienko and N. Andrienko. Interactive spatiotemporal cluster analysis of vast challenge 2008 datasets. In VAKD '09: Proceedings of the ACM SIGKDD Workshop on Visual Analytics and Knowledge Discovery, pages 5-11, New York, NY, USA, 2009. ACM.

[2] G. Andrienko, N. Andrienko, and S. Wrobel. Visual analytics tools for analysis of movement data. SIGKDD Explor. Newsl., 9(2):38-46, 2007.

[3] P. Bak, F.Mansmann, H. Janetzko, and D. Keim. Spatiotemporal analysis of sensor logs using growth ring maps. IEEE Transactions on Visualization and Computer Graphics, 15:913-920, 2009.

[4] S. Bitgood and S. Lankford. Museum orientation and circulation. Visitor Behavior, 15:4-6, 1995.

[5] K. Börner and S. Penumarthy. Social diffusion patterns in threedimensiona virtual worlds. Information Visualization, 2(3):182-198, 2003.

[6] F. Brian and J. Pritchard. Visualization of historical events using lexis pencils. in case studies of visualization in the social sciences, 1997. http://www.cas.lancs.ac.uk/alcd/visual.

[7] J. V. Carlis and J. A. Konstan. Interactive visualization of serial periodic data. In UIST '98: Proceedings of the 11th annual ACM symposium on User interface software and technology, pages 29-38, New York, NY, USA, 1998. ACM.

[8] L. Chittaro, R. Ranon, and L. Ieronutti. Vu-flow: A visualization tool for analyzing navigation in virtual environments. IEEE Transactions on Visualization and Computer Graphics, 12:1475-1485, 2006.

[9] P. Coulton, W. Bamford, K. Cheverst, and O. Rashid. 3d space-time visualization of player behaviour in pervasive location-based games. Int. J. Comput. Games Technol., 2008:1-5, 2008.

[10] T. Crnovrsanin, C. Muelder, C. Correa, and K.-L. Ma. Proximitybased visualization of movement trace data. In Proceedings of IEEE Symposium on Visual Analytics Science and Technology (VAST 2009), October 2009.

[11] P. N. Dixit and G. M. Youngblood. Optimal information placement in an interactive 3d environment. In Sandbox '07: Proceedings of the 2007 ACMSIGGRAPH symposium on Video games, pages 141-147, New York, NY, USA, 2007. ACM.

[12] P. Dourish and M. Chalmers. Running out of space: Models of information navigation. In HCI, Glasgow, August 1994.

[13] M. Harrower and C. Brewer. Colorbrewer.org: an online tool for selecting colour schemes for maps. Cartographic Journal, 40(1):2737, 2003.

[14] http://www.cs.umd.edu/hci/VASTchallenge08/. IEEE vast 2008 challenge, 2008.

[15] T. Kanda, M. Shiomi, L. Perrin, T. Nomura, H. Ishiguro, and N. Hagita. Analysis of people trajectories with ubiquitous sensors in a science museum. In IEEE International Conference on Robotics and Automation (ICRA2007), pages 4846-4853, 2007.

[16] I. R. Kaynar, S. Psarra, and J. Wineman. Experiencing museum gallery layouts through local and global visibility properties in morphology: an inquiry on the ycba, the moma and the hma. In Proc. 7th International Space Syntax Symposium, Stockholm, Sweden, June 2009.

[17] R.Maciejewski, S. Rudolph, R. Hafen, A. Abusalah, M. Yakout, M. Ouzzani, W. Cleveland, S. Grannis, and D. Ebert. A visual analytics approach to understanding spatiotemporal hotspots. Visualization and Computer Graphics, IEEE Transactions on, 16(2):205 -220, marchapril 2010.

[18] J. Oda and R. Thawonmas. Comparison of user trajectories based on coordinate data and state transitions. In Intelligent Information Hiding and Multimedia Signal Processing, 2009. IIH-MSP '09. Fifth International Conference on, pages 1134-1137, sept. 2009.

[19] K. Sookhanaphibarn and R. Thawonmas, "Digital Museums in 3D Virtual Environments," Handbook of Research on Methods and Techniques for Studying Virtual Communities: Paradigms and Phenomena, IGI Global Publisher, 2011, pp. 713-730.

[20] Kingkarn Sookhanaphibarn, Ruck Thawonmas, and Frank Rinaldo, "Visualization of Visitor Circulation in Arts and Cultural Exhibition," Abstracts of Digital Humanities 2011 (DH2011), Stanford University, CA, USA, pp. 365-368, Jun. 19-22, 2011.

[21] J. B. Tenenbaum, V. de Silva, J. C. Langford (2000). A global geometric framework for nonlinear dimensionality reduction. Science 290 (5500): 2319-2323, 22 December 2000.

[22] C. Tominski, J. Abello, and H. Schumann. Axes-based visualizations with radial layouts. In Proceedings of the 2004 ACM symposium on Applied computing, pages 1242 - 1247, 2004.

[23] C. Tominski, P. Schulze-Wollgast, and H. Schumann. 3d information visualization for time dependent data on maps. In IV '05: Proceedings of the Ninth International Conference on Information Visualisation, pages 175-181, Washington, DC, USA, 2005. IEEE Computer Society.

[24] E. Veron and M. Levasseur. Bibliothque publique d'information: Centre georges pompidou. In Proceedings of the Ninth International Conference on Information Visualisation, Paris, 1983. 


\section{BIOGRAPHIES}
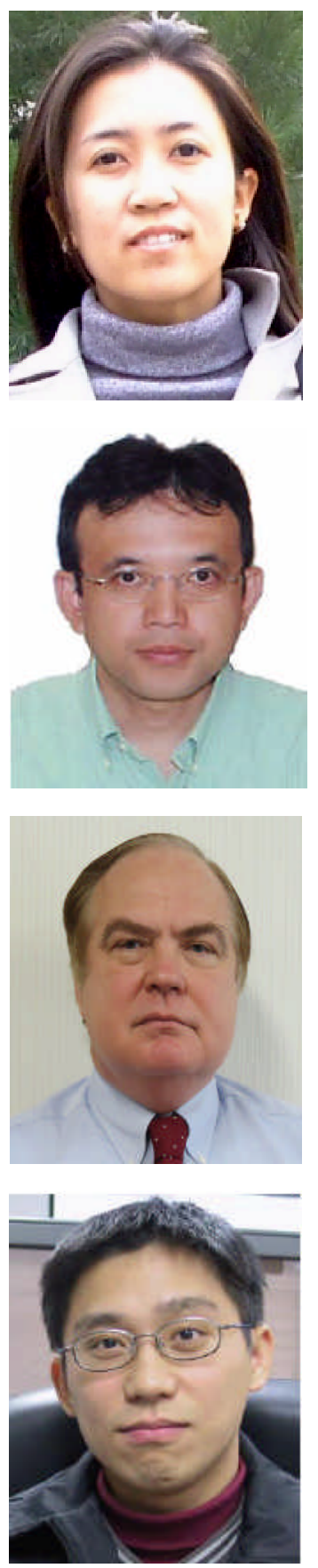

Kingkarn Sookhanaphibarn ${ }^{1}$ received a B.Sc. in Computer Science from Chulalongkorn University, Thailand in 1997, a M.Sc. in Computational Science from Chulalongkorn University in 1999, and a Ph.D. in Computer Science from Chulalongkorn University in 2006. During 2000-2004, he was a recipient of the scholarship from the Royal Golden Jubilee Ph.D. (RGJ-Ph.D.) Program under the Thailand. Research Fund.

After receiving her $\mathrm{PhD}$, Dr. Sookhanaphibarn was a lecture in Department of Imaging Technology Chulalongkorn University for three years. From 2009 to present, she has joined the GCOE Program for Digital Humanities of Japanese Arts and Cultures in Ritsumeikan University as a Post-Doctoral fellow. Her research interests include information visualization, user modeling and human-computer interaction, and artificial intelligence.

Email: kingkarns@gmail.com

Ruck Thawonmas received B.Eng. in Electrical Engineering from Chulalongkorn University, Thailand, in 1987, M.Eng. in Information Science from Ibaraki University, Japan, in 1990, and D.Eng. in Information Engineering from Tohoku University, Japan, in 1994. During 1987-1993, he was a recipient of the Japanese Government (Monbusho) Scholarship.

Since April 2002, Dr. Thawonmas has been at Ritsumeikan University in Japan and has become a full professor in the Department of Human and Computer Intelligence since April 2004. Prior to that, he was with Hitachi, Ltd., RIKEN, University of Aizu, and Kochi University of Technology. His research interests include online games, story generation, and entertainment computing. He is a senior member of IEEE.

Email: ruck@ci.ritsumei.ac.jp

Frank Rinaldo received a BS in Mathematics and Physics from the Illinois Institute of Technology in 1971, a MS in Computer Science from DePaul University in 1981, a MBA in International Business is 1983, and a PhD in Computer Science from the Illinois Institute of Technology in 1989.

Since April 2003, Dr. Rinaldo has been a Professor in the Department of Human and Computer Intelligence at Ritsumeikan University in Japan. Prior to that, he was CEO of a computer game development company. His research interests include: online games, strategy games, game artificial intelligence, parallel processing, distributed computing, natural language processing, genetic algorithms and swarm computing.

Email: rinaldo@is.ritsumei.ac.jp

Kuan-Ta Chen (also known as Chun-Yang Chen) received his B.S. and M.S. in Computer Science from National Tsing Hua University in 1998 and 2000, respectively. He received his Ph.D. in Electrical Engineering from National Taiwan University in 2006.

Prior to taking his academic path, Dr. Chen was active as a programmer specialized in Windows and system programming, a technical writer of four books, a technical lecturer of programming courses, and a shareware developer.

Recently, Dr. Chen has been at Institute of Information Science, Academia Sinica, as an Associate Research Fellow. He also works as a Joint Appointed Associate Research Fellow at Research Center for Information Technology Innovation and the Director of Multimedia Networking and Systems Lab.

His research interests include Internet \& Multimedia Quality of Experience (QoE) management, Internet measurement, network security, and online games. Much of his recent work focused on QoE-aware multimedia system design, AI-hard problem solving using human computation, and the combination of both directions. He received the Best Paper Award (with Ieng-Fat Lam and Ling-Jyh Chen) in IWSEC 2008 and K. T. Li . Distinguished Young Scholar Award from ACM Taipei/Taiwan Chapter in 2009. He also received the Outstanding Young Electrical Engineer Award from The Chinese Institute of Electrical Engineering in 2010. He is a member of ACM, IEEE, IICM, and CCISA.

Email: ktchen@iis.sinica.edu.tw

\section{Copyright (C) 2013 by IPI Press Special permissions granted authors at www.ijvr.org/Copyrights.html}

